\title{
Do statin users adhere to a healthy diet and lifestyle? The Australian Diabetes, Obesity and Lifestyle Study
}

\author{
Simran Johal ${ }^{1,2}$, Kris M Jamsen ${ }^{1}$, J Simon Bell ${ }^{1}$, Kevin P McNamara ${ }^{1,3}$, Dianna J Magliano ${ }^{4}$, \\ Danny Liew ${ }^{5}$, Taliesin E Ryan-Atwood ${ }^{1}$, Claire Anderson ${ }^{2}$, Jenni Ilomäki ${ }^{1,5}$
}

1. Centre for Medicine Use and Safety, Faculty of Pharmacy and Pharmaceutical Sciences, Monash University, Melbourne, Australia

2. Division of Pharmacy Practice \& Policy, School of Pharmacy, University of Nottingham, Nottingham, United Kingdom

3. Greater Green Triangle University Department of Rural Health, Flinders University and Deakin University, Warrnambool, Australia

4. Baker IDI Heart and Diabetes Institute, Melbourne, Australia

5. Department of Epidemiology and Preventive Medicine, Monash University, Melbourne, Australia

\section{Address for correspondence:}

Dr Jenni Ilomäki

Centre for Medicine Use and Safety

Faculty of Pharmacy and Pharmaceutical Sciences

Monash University

Melbourne, Australia

Phone: +61 399039554

Email: jenni.ilomaki@monash.edu

Word Count - 4400 


\section{Abstract}

Background: Lifestyle and dietary advice typically precedes or accompanies the prescription of statin medications. However, evidence for adherence to this advice is sparse. The objective was to compare saturated fat intake, exercise, alcohol consumption and smoking between statin users and non-users in Australia.

Methods: Data were analyzed for 4,614 participants aged $\geq 37$ years in the Australian Diabetes, Obesity and Lifestyle study in 2011-2012. Statin use, smoking status and physical activity were self-reported. Saturated fat and alcohol intake were measured via a food frequency questionnaire. Multinomial Logistic regression was used to compute adjusted odds ratios (ORs) and 95\% confidence intervals (CIs) for the associations between statin use and the four lifestyle factors. All models were adjusted for age, sex, education, number of GP visits, body mass index, hypertension, diabetes and prior cardiovascular diseases.

Results: In total 1108 (24\%) participants used a statin. Statin users were $29 \%$ less likely to be within the highest quartile vs. the lowest quartile of daily saturated fat intake compared to non-users (OR $0.71,95 \% \mathrm{CI} 0.54-0.94)$. There were no statistically significant associations between statin use and smoking, physical activity or alcohol consumption.

Conclusions: Smoking status, alcohol consumption and exercise level did not differ between users and non-users of statins. However, statin users were less likely to consume high levels of saturated fat than non-users. We found no evidence that people took stains to compensate for a poor diet or lifestyle.

\section{Abstract word count: 232}

Key Words: smoking, alcohol drinking, exercise, diet, HMG-CoA reductase inhibitors, epidemiology, cohort study 


\section{Introduction}

Lifestyle and dietary modifications are the cornerstone of primary and secondary prevention of cardiovascular disease (CVD). Smoking cessation, low-moderate alcohol consumption, exercise and reduction in saturated fat intake reduce CVD risk. ${ }^{1-4}$ Guidelines recommend that lifestyle and dietary advice should precede or accompany the prescription of statin medications. ${ }^{5,6}$ However, as lifestyle and dietary modifications can be challenging to implement and maintain, guidelines may not be followed. ${ }^{7,8}$

Meta-analyses have demonstrated the effectiveness of statin treatment in reducing cardiovascular-related mortality and morbidity. ${ }^{9,} 10$ The prescribing of statins has increased rapidly in Australia, Europe and the US. The prevalence of statin use increased from $17 \%$ in 2001 to $35 \%$ in 2012 among Australian veterans. ${ }^{11}$ In the UK, the threshold for prescribing statins for primary prevention has been lowered from a 20\% 10-year CVD risk to $10 \% 10$ year CVD risk. ${ }^{12}$ New US guidelines suggest statins be prescribed to those with a $7.5 \% 10$ year risk. ${ }^{13}$ This corresponds to an estimated additional 13 million individuals being eligible for statin therapy. ${ }^{13}$

It is not known whether statin use complements or substitutes a healthy lifestyle and diet. Patients may perceive that statins represent an alternative path to health benefits, ${ }^{7}$ and prescribing of statins may diminish clinicians' focus on CVD risk reduction via lifestyle and diet. ${ }^{14}$ Previous studies comparing lifestyle factors between statin users and non-users have reported inconsistent findings. A Swedish study in 2012 reported statin users were more likely to understand the importance of CVD risk reduction and were more likely to undertake lifestyle modifications. ${ }^{15}$ However, more recent US studies have reported that statin users had lower exercise levels ${ }^{16}$, and greater increases in caloric and fat intake than non-users of statins. ${ }^{17}$ These findings may be explained by risk compensation, in which people receiving a particular risk-lowering intervention are more likely to engage in risky behaviors. ${ }^{18}$ 
Alternatively, statins may cause muscular pain that discourages exercise or be prescribed to patients with comorbidities that prevent participation in exercise. ${ }^{16,19}$

No population-based studies on the association between statin use and lifestyle or diet have been conducted in Australia. The objective of this study was to compare saturated fat intake, exercise, alcohol consumption and smoking between statin users and non-users in Australia.

\section{Methods}

Study design, setting and participants

The Australian Diabetes, Obesity and Lifestyle Study (AusDiab) is a 12-year longitudinal population based study. The baseline assessment was conducted 1999-2000, during which a total of 11,247 people aged 25 years and older in 42 randomly selected urban and rural areas across seven states and territories consented to participate. Data on clinical, demographic, health and lifestyle factors were collected using a series of interviewer and self-administered questionnaires with biomedical examinations conducted at local testing sites. Two follow-up examinations were conducted. The first follow-up took place in 2004-2005 (wave 2) and $6,400(59.3 \%)$ of eligible participants from baseline participated. The second follow-up took place in 2011-2012 (wave 3) and 4,614 (44.6\%) of eligible participants from the baseline assessment participated. The full study methods and response rate for the baseline survey have been described previously. ${ }^{20}$ The current study was a cross sectional analysis of data collected in 2011-2012 (wave 3).

\section{Definitions and Measurements}

Medication use was self-reported by participants using a self-administered questionnaire. All medications were coded using the Anatomical Therapeutic Chemical (ATC) classification recommended by the World Health Organization (WHO) ${ }^{21}$ Statin users were defined as those 
who took a statin (ATC codes C10AA, C10BA, C10BX) for at least 2 weeks within the last 3 months.

The lifestyle factors measured as outcomes were smoking status, alcohol consumption, exercise level and saturated fat intake. Smoking status was measured via self-administered questionnaire and participants were categorized as current smokers, former smokers or never smokers. Current smokers included those who smoked on a daily basis, former smokers included those who smoked less than daily in the past 3 months, and never smokers were defined who smoked less than 100 cigarettes during their lifetime. This categorization was consistent with previous research. ${ }^{20}$

Physical activity was measured via a self-administered questionnaire using questions regarding time spent doing vigorous and moderate physical activity in the previous week. Physical activity was categorized consistent with previous research, ${ }^{20}$ into sedentary (no participation in physical activity in the previous week), insufficiently active (>0-149 minutes of moderate activity in the previous week and >0-74 minutes of vigorous activity in the previous week) and sufficiently active ( $\geq 150$ minutes of moderate-intensity or $\geq 75$ minutes of vigorous activity in the previous week).

Saturated fat intake as grams per day was calculated using a self-administered food frequency questionnaire (FFQ), recalling intake over the previous 12 months. ${ }^{20}$ Saturated fat intake was expressed as a percentage of total daily energy intake and categorized into quartiles. ${ }^{22}$ Daily alcohol consumption was obtained via the FFQ and expressed in grams of ethanol per day. Daily alcohol consumption was categorized into no drinking $(0 \mathrm{~g})$, low-moderate drinking $(>0-20 \mathrm{~g})$, heavy drinking $(>20-40 \mathrm{~g})$ and excessive drinking $(>40 \mathrm{~g})$, where $10 \mathrm{~g}$ of alcohol is equivalent to 1 unit of alcohol. ${ }^{4,23}$ 


\section{Covariates}

Age, sex, education level, number of general practitioner (GP) visits, body mass index (BMI), hypertension, diabetes and prior CVD were considered as covariates. Participant's education level was assessed at baseline and wave 2. If a participant reported a higher level of education at wave 2 then this level was used to replace their baseline response. Participants were categorized into having not attended school, some primary or secondary education including completion of secondary education vs. having some education above secondary level. The number of GP visits within the last three months was obtained via an interviewer administered questionnaire. This variable was categorized into 0 visits, 1 visit, 2 visits and $\geq 3$ visits. BMI $\left(\mathrm{kg} / \mathrm{m}^{2}\right)$ was calculated during biomedical examinations and was categorized into normal (BMI <25.0), overweight (BMI 25.0-29.9) and obese (BMI $\geq 30.0) .{ }^{24}$ Hypertension was defined based on blood pressure measurement and participant's self-report of medications used for treating hypertension. Blood pressure (BP) measurements were completed in a seated position using an automated blood pressure monitor which was regularly calibrated (Dinamap® Pro-series Monitor Model DP 101- NIBP, Pulse and recorder, GE Medical Systems Information Technologies, Milwaukee, USA). Three measurements were taken at 30 second intervals. The mean of the first two measurements were taken unless the difference between the first and second measurement was greater than $10 \mathrm{mmHg}$. In this case the third measurement was considered, and the mean of the two closest readings was used. Hypertension was categorized as normotension (BP <140/90 $\mathrm{mmHg}$ and no medication use for hypertension), untreated hypertension ( $\mathrm{BP} \geq 140 / 90 \mathrm{mmHg}$ and no medication use for hypertension) and treated hypertension (those who reported taking medications for hypertension). ${ }^{25}$

Participants were considered to have diabetes if their fasting blood glucose was $\geq 7.0 \mathrm{mmol} / \mathrm{L}$, two-hour blood glucose was $\geq 11.1 \mathrm{mmol} / \mathrm{L}$, or they reported using medications for diabetes 
(ATC code A10). ${ }^{20,26}$ Participants were regarded to have prior CVD if they self-reported having had angina, stroke or myocardial infarction (MI) at baseline, had a MI, stroke, coronary artery angioplasty surgery or coronary artery bypass surgery between baseline and wave 3, or reported using nitrates (ATC code C10DA) at wave 3.

\section{Statistical Analysis}

Demographic and clinical characteristics of statin users and non-users were presented as proportions or means with standard deviations. The association between statin use and each lifestyle factor was examined using multinomial logistic regression. One regression model was computed for each of the four lifestyle factors. For each model, statin use (yes/no) was the primary exposure variable and age, sex, hypertension, diabetes, BMI, number of GP visits in the previous 3 months, prior CVD and lifestyle factors other than the outcome were treated as covariates. Odds ratios (ORs) and $95 \%$ confidence intervals (CIs) were calculated. The analyses were performed using SAS (version 9.4, Cary, NC).

To investigate the representativeness of participants at wave 3 of the original cohort at baseline we compared baseline characteristics between those participants who attended and those who did not attend the wave 3 examination. The proportion on females was similar between the groups, but participants who attended wave 3 were younger (mean age 48.9 years vs. 53.7 years). Attendees had on average less GP visits during the previous 12 months, the proportion of people with hypertension, diabetes and prior cardiovascular disease were lower and they had lower baseline prevalence of lipid lowering medicine use than those who did not attend. 


\section{Results}

A total of 1108 of the 4614 participants (24\%) in wave 3 used a statin. The mean age of statin users was 67.8 years compared to 58.6 years in non-users. Half of all statin users were male (51.3\%) compared to only $42.6 \%$ of non-users. The prevalence of prior CVD, diabetes and treated hypertension was higher among statin users compared to non-users (corresponding comparisons of $21.7 \%$ vs $2.7 \%, 25.2 \%$ vs. $5.1 \%$ and 57.2 vs. $21.3 \%$ respectively). The characteristics of statin users and non-users are summarized in Table 1. The prevalence of current smoking was lower in statin users than non-users $(3.8 \%$ in statin users, $6.3 \%$ in nonusers).

After adjustment for the covariates, statin use was not associated with being in the second $(\mathrm{OR}=1.05 ; 95 \% \mathrm{CI}=0.82-1.35)$ or third $(\mathrm{OR}=0.80 ; 95 \% \mathrm{CI}=0.62-1.04)$ quartile vs. the lowest quartile of daily saturated fat intake (Table 2). However, statin users were $29 \%$ less likely to be within the highest quartile vs. the lowest quartile of daily saturated fat intake compared to non-users $(\mathrm{OR}=0.71,95 \% \mathrm{CI}=0.54-0.94)$. There was no statistically significant associations between statin use and smoking, physical activity or alcohol consumption.

\section{Discussion}

The main finding of this study was that statin users were $29 \%$ less likely consume high amounts of saturated fat as a percentage of total daily energy intake compared to non-users. Statin users and non-users were equally likely to consume high levels of alcohol and have sedentary lifestyle. Similarly, there was no difference in current smoking and former smoking, when adjusting for clinically relevant covariates.

Our study found statin users had lower saturated fat intake compared to non-users. This is consistent with a previous study in Sweden which found statin users had better dietary habits 
compared to non-users. ${ }^{15}$ Statin users may be more exposed to dietary information from their healthcare professionals. However, another US study showed that caloric and fat intake among statin users increased by $9.6 \%$ over a 10 year period among the US general population while in non-users the intake remained constant over the same period. ${ }^{17}$ This suggest that the attitudes towards dietary recommendations may change over time, where people prescribed statins increasingly accept statins as a compensatory measure for an unhealthy diet. It is unclear whether the lifestyle behaviors of statin users in the US are generalizable to Australia. Unlike the US repeated cross-sectional study, our study was single cross-sectional study and, therefore, we were unable to assess whether lifestyle factors changed over time. Further longitudinal research is needed to determine whether possible lifestyle modifications among statin users in Australia are sustained over time.

Furthermore, the inconsistencies between Swedish, US and Australian research suggest that statin users in these countries may have a different lifestyle profile. ${ }^{15,17}$ This is an important consideration for extrapolating the findings of research that has investigated the cardiovascular outcomes of statin users in these countries.

In the present study, smoking status did not differ between statin users and non-users in the adjusted analyses with low rates of current smokers in both groups $(3.8 \%$ and $6.3 \%$ respectively). The finding is consistent with previous literature where people have been observed to continue smoking despite known negative health effects. ${ }^{27}$ Barriers associated with smoking cessation range from reliance on smoking for stress management, not seeking adequate professional help, ${ }^{28}$ fear of weight gain and concerns regarding costs, particularly in vulnerable groups. ${ }^{27}$ Moreover, a significant proportion of non-smokers with CHD who are treated with statins are exposed to passive smoking, which may hinder smoking cessation. ${ }^{29}$, ${ }^{30}$ One study found that GP's only initiated discussion regarding smoking cessation when patients presented with smoking related symptoms. ${ }^{33}$ Physicians perceived discussions were 
too time consuming. Lack of support from physicians and lack of patients' awareness of cardiovascular risk factors may be a barrier to healthy lifestyle changes. ${ }^{31,32}$

Moreover, a review of several landmark trials observed that smoking diminishes the benefits of statins in reducing CVD risk. ${ }^{34}$ The review found that the risk of a CVD event in untreated non-smokers was of the same magnitude to the risk of smokers taking statins. Therefore, although the prevalence of current smoking in the present study was low, the importance of smoking cessation may need to be re-iterated to smokers who are prescribed statins.

This study showed no association between statin use and physical activity. It is possible that statin users may have exercised less than non-users prior to statin initiation or that statin-users may have conditions which inhibit their ability to exercise. Once prescribed statins, people may have increased their exercise levels to the same level as non-users however further longitudinal research is needed. Previous studies have found that statin users undertake less exercise than non-users. ${ }^{16}$ One possible explanation for these findings may be due to statin related side effects ranging from rare cases of rhabdomyolysis to more common muscle weakness and muscle pain. ${ }^{35}$ A study in France found among 7,924 patients taking statins, $11 \%$ experienced muscle symptoms, $4 \%$ had symptoms severe enough to interfere with daily activities, and $0.4 \%$ were confined to bed with their symptoms. ${ }^{36}$

No difference in alcohol consumption between statin users and non-users was found. There is no consensus on the effect of statin use on alcohol consumption as previous studies have produced conflicting results. A recent study reported that statin use was associated with a $15 \%$ increase in moderate alcohol use among men, ${ }^{37}$ however other studies have observed lower alcohol consumption among statin users. ${ }^{38}$ Only $4 \%$ of GPs surveyed reported asking patients about their alcohol consumption 'all of the time, ${ }^{39}$ which may reduce patient awareness about the effects of alcohol. Low-moderate alcohol consumption of up to two standard drinks daily has been shown to be the most beneficial in reducing CVD risk. ${ }^{40}$ 
Despite this, non-drinkers are not advised to start drinking to reduce their CVD risk. ${ }^{41} \mathrm{~A}$ meta-analysis of brief interventions in primary care aimed at reducing alcohol consumption demonstrated a decline of $38 \mathrm{~g}$ of ethanol per week following intervention. ${ }^{42}$ However, this intervention may be underutilized due to healthcare workers having insufficient time or lack of training ${ }^{39}$ which may have implications for excessive drinkers, who could potentially benefit from such service.

The intensity of preventative actions recommended by clinicians may have been linked to participants' total cardiovascular risk. It is possible that prescribers of statins were more proactive in managing a range of cardiovascular risk factors, including smoking, diet, lifestyle and diabetes. We are unable to determine what extent statin users and non-users may have received different advice from their clinicians, and to what extent this advice was followed by patients. Our study was cross-sectional in design and, therefore, it was not possible to determine whether statin users had poorer smoking, dietary and lifestyle behaviors prior to statin initiation.

\section{Strengths and limitations}

This study has several strengths. The data consisted of a random sample of more than 4600 participants from urban suburban and regional areas across Australia. The data were collected in 2011-2012 and reflect relatively recent Australian prescribing and lifestyle patterns. To ensure a representative sample stratified cluster sampling was employed. Multivariate analyses were performed to accommodate potential confounding factors.

There are some limitations to the present study. Self-reporting of statin use and lifestyle factors may have caused some participants to incorrectly report their statin use or lifestyle. This may have resulted in over- or under-estimation of the effect of statin use or the covariates. The participation rate at wave three was 4614 of the 11,247 who consented to 
participate at baseline. Although we utilized the most recent data collected, participants at wave three might not be a representative sample of those at baseline. We compared the baseline characteristics of those who participated to those who did not participate at wave three. This shows that participants were less likely to be... Missing data in this analysis were excluded. Although the number of missing values per variable was relatively small $(<12 \%)$, this might have led to partially biased estimates in our multivariate analyses. For example, if food frequency diary was not completed for those who were excessive alcohol consumers or had high saturated fat intake, this might have caused underestimation of these parameters. The cross-sectional design did not allow an assessment of causality related to statin use and lifestyle factors. This also means that we did not have information on statin initiation and how lifestyle factors may have changed over time.

\section{Conclusion}

Statin users had lower saturated fat intake than non-users. However, there was no difference in smoking status, exercise levels or alcohol consumption between statin users and non-users after adjusting for clinically important covariates. We found no evidence that people take statins to compensate a healthy lifestyle. Our results suggest that statin users in Australia may have a different lifestyle profile to statin users in the U.S. and Sweden. Longitudinal studies are needed to whether possible lifestyle modifications among statin users in Australia are sustained over time.

\section{Author Contributions}

SJ, KJ, JB, KM, DM, DL and JI contributed to the conception or design of the work. SJ, KJ, JB, KM, DM, DL, TRA, CA and JI contributed to the acquisition, analysis, or interpretation of data for the work. SJ drafted the manuscript. SJ, KJ, JB, KM, DM, DL, TRA, CA and JI 
critically revised the manuscript. All gave final approval and agree to be accountable for all aspects of work ensuring integrity and accuracy.

\section{Declaration of conflicting interests}

The authors declare that there is no conflict of interest.

\section{Funding}

KJ was supported by the National Health and Medical Research Council Cognitive Decline Partnership Centre. KM was supported by a Heart Foundation postdoctoral award (grant number 100187). JI was supported by a National Health and Medical Research Council Fellowship (grant number 1072137). 


\section{REFERENCES}

1. Sarikaya H, Ferro J and Arnold M. Stroke prevention - medical and lifestyle measures. European neurology. 2015; 73: 150-7.

2. Flock MR and Kris-Etherton PM. Dietary guidelines for Americans 2010: Implications for cardiovascular disease. Current atherosclerosis reports. 2011; 13: 499-507.

3. Sattelmair J, Pertman J, Ding EL, Kohl HW, Haskell W and Lee IM. Dose-response between physical activity and risk of coronary heart disease: A meta-analysis. Circulation. 2011; 124: 789-95.

4. Ronksley PE, Brien SE, Turner BJ, Mukamal KJ and Ghali WA. Association of alcohol consumption with selected cardiovascular disease outcomes: A systematic review and meta-analysis. BMJ. 2011; 342.

5. Perk J, De Backer G, Gohlke H, et al. European guidelines on cardiovascular disease prevention in clinical practice (version 2012). The fifth joint task force of the european society of cardiology and other societies on cardiovascular disease prevention in clinical practice (constituted by representatives of nine societies and by invited experts). European heart journal. 2012; 33: 1635701.

6. Reiner Z, Catapano AL, De Backer G, et al. Esc/eas guidelines for the management of dyslipidaemias: The task force for the management of dyslipidaemias of the european society of cardiology (esc) and the european atherosclerosis society (eas). European heart journal. 2011; 32: 1769-818.

7. LaRosa JC. Means and ends of statins and low-density lipoprotein cholesterol lowering. Journal of the American College of Cardiology. 2007; 50: 419-20.

8. King DE, Mainous lii AG, Carnemolla M and Everett CJ. Adherence to healthy lifestyle habits in US adults, 1988-2006. Am J Med. 2009; 122: 528-34.

9. Baigent C, Keech A, Kearney PM, et al. Efficacy and safety of cholesterol-lowering treatment: Prospective meta-analysis of data from 90,056 participants in 14 randomised trials of statins. Lancet. 2005; 366: 1267-78.

10. LaRosa JC, He J and Vupputuri S. Effect of statins on risk of coronary disease: A meta-analysis of randomized controlled trials. Jama. 1999; 282: 2340-6.

11. Gadzhanova S, Roughead E and Ilomäki J. Use of statins in an Australian elderly population. Int J Clin Cardiol. 2014; 1: 1-4.

12. National Institute for Health and Care Excellence. Cardiovascular disease: Risk assessment and reduction, including lipid modification, http://www.nice.org.uk/guidance/cg181 (2014, Accessed 14 April 2015)

13. Pencina MJ, Navar-Boggan AM, D'Agostino RB, et al. Application of new cholesterol guidelines to a population-based sample. N Engl J Med. 2014; 370: 1422-31.

14. Mascitelli $L$ and Pezzetta F. Statins for primary prevention of coronary artery disease. Lancet. 2007; 369: 1078-9.

15. Lytsy P, Burell G and Westerling R. Cardiovascular risk factor assessments and health behaviours in patients using statins compared to a non-treated population. International journal of behavioral medicine. 2012; 19: 134-42.

16. Lee $D H$, Markwardt $S$, Goeres $L$ and et al. Statins and physical activity in older men: The osteoporotic fractures in men study. JAMA Intern Med. 2014; 174: 1263-70.

17. Sugiyama T, Tsugawa $Y$, Tseng $C$, Kobayashi $Y$ and Shapiro MF. Different time trends of caloric and fat intake between statin users and nonusers among us adults: Gluttony in the time of statins? JAMA Intern Med. 2014; 174: 1038-45.

18. Brewer NT, Cuite CL, Herrington JE and Weinstein ND. Risk compensation and vaccination: Can getting vaccinated cause people to engage in risky behaviors? Annals of behavioral medicine : a publication of the Society of Behavioral Medicine. 2007; 34: 95-9.

19. Simic I and Reiner Z. Adverse effects of statins - myths and reality. Current pharmaceutical design. 2015; 21: 1220-6. 
20. Dunstan DW, Zimmet PZ, Welborn TA, et al. The Australian diabetes, obesity and lifestyle study (AusDiab) -methods and response rates. Diabetes Res Clin Pract. 2002; 57: 119-29.

21. Organization WH. ATC/DDD index 2015, http://www.whocc.no/atc_ddd_index/ (2015, Accessed 14 April 2015)

22. Puaschitz NG, Strand E, Norekval TM, et al. Dietary intake of saturated fat is not associated with risk of coronary events or mortality in patients with established coronary artery disease. The Journal of nutrition. 2015; 145: 299-305.

23. National Health and Medical Research Council. Australian guidelines to reduce health risks from drinking alcohol. In: National Health and Medical Research Council (NHMRC), (ed.). Canberra: Australian Government, 2009.

24. World Health Organization Bmi classification

http://apps.who.int/bmi/index.jsp?introPage=intro_3.html (2015, Accessed 14 April 2015)

25. World Health Organization and International Society of Hypertension Writing Group. 2003 world health organization (WHO)/international society of hypertension (ish) statement on management of hypertension. J Hypertens. 2003; 21: 1983-92.

26. World Health Organization. Definition, diagnosis and classification of diabetes mellitus and its complications, http://apps.who.int/iris/bitstream/10665/66040/1/WHO_NCD_NCS_99.2.pdf (1999, Accessed 14 April 2015)

27. Rosenthal L, Carroll-Scott A, Earnshaw VA, et al. Targeting cessation: Understanding barriers and motivations to quitting among urban adult daily tobacco smokers. Addictive behaviors. 2013; 38: 1639-42.

28. Twyman L, Bonevski B, Paul C and Bryant J. Perceived barriers to smoking cessation in selected vulnerable groups: A systematic review of the qualitative and quantitative literature. $B M J$ open. 2014; 4: e006414.

29. Prugger C, Wellmann J, Heidrich J, et al. Readiness for smoking cessation in coronary heart disease patients across europe: Results from the euroaspire iii survey. European journal of preventive cardiology. 2015; 22: 1212-9.

30. Prugger C, Wellmann J, Heidrich J, et al. Passive smoking and smoking cessation among patients with coronary heart disease across europe: Results from the euroaspire iii survey. European heart journal. 2014; 35: 590-8.

31. Reiner Z, Sonicki Z and Tedeschi-Reiner E. Physicians' perception, knowledge and awareness of cardiovascular risk factors and adherence to prevention guidelines: The percro-doc survey. Atherosclerosis. 2010; 213: 598-603.

32. Reiner Z, Sonicki Z and Tedeschi-Reiner E. Public perceptions of cardiovascular risk factors in croatia: The percro survey. Preventive medicine. 2010; 51: 494-6.

33. Helgason AR and Lund KE. General practitioners' perceived barriers to smoking cessationresults from four Nordic countries. Scandinavian journal of public health. 2002; 30: 141-7.

34. Milionis HJ, Rizos E and Mikhailidis DP. Smoking diminishes the beneficial effect of statins: Observations from the landmark trials. Angiology. 2001; 52: 575-87.

35. Thompson PD and Parker B. Statins, exercise, and exercise training. Journal of the American College of Cardiology. 2013; 62: 715-6.

36. Bruckert E, Hayem G, Dejager S, Yau C and Begaud B. Mild to moderate muscular symptoms with high-dosage statin therapy in hyperlipidemic patients--the PRIMO study. Cardiovascular drugs and therapy / sponsored by the International Society of Cardiovascular Pharmacotherapy. 2005; 19: 403-14.

37. Kaestner R, Darden $M$ and Lakdawalla $D$. Are investments in disease prevention complements? The case of statins and health behaviors. J Health Econ. 2014; 36: 151-63.

38. Lipworth L, Fazio S, Kabagambe EK, et al. A prospective study of statin use and mortality among 67,385 blacks and whites in the southeastern United States. Clin Epidemiol. 2014; 6: 15-25. 
39. Kaner EF, Heather N, McAvoy BR, Lock CA and Gilvarry E. Intervention for excessive alcohol consumption in primary health care: Attitudes and practices of English general practitioners. Alcohol Alcohol. 1999; 34: 559-66.

40. Rimm EB, Williams P, Fosher K, Criqui M and Stampfer MJ. Moderate alcohol intake and lower risk of coronary heart disease: Meta-analysis of effects on lipids and haemostatic factors. BMJ. 1999; 319: 1523-8.

41. Movva R, Figueredo V.M. Alcohol and the heart: To abstain or not to abstain? Int J Cardiol. 2013; 164(3):267-76.

42. Bertholet N, Daeppen J, Wietlisbach V, Fleming M and Burnand B. Reduction of alcohol consumption by brief alcohol intervention in primary care: Systematic review and meta-analysis. Arch Intern Med. 2005; 165: 986-95. 